\title{
Perioperative Management of Alcohol Withdrawal Syndrome
}

\author{
Alexander Lavinius Ungur ${ }^{\mathrm{a}}$ Tim Neumann $^{\mathrm{b}} \quad$ Friedrich Borchers $^{\mathrm{a}}$ \\ Claudia Spies $^{a}$ \\ ${ }^{a}$ Department of Anesthesiology and Intensive Care Medicine, Campus Charité Mitte and Campus Virchow-Klinikum, \\ Charité - Universitätsmedizin Berlin, Berlin, Germany; ${ }^{\mathrm{b}}$ Department of Anesthesiology and Intensive Care Medicine, \\ Campus Benjamin Franklin, Charité - Universitätsmedizin Berlin, Berlin, Germany
}

\section{Keywords}

Alcohol withdrawal syndrome $\cdot$ Alcohol $\cdot$ Ethanol $\cdot$ Alcohol withdrawal · Delirium

\begin{abstract}
Background: In the perioperative course, alcohol withdrawal syndrome (AWS) can occur in any setting, especially in aero-digestive and acute trauma surgery. Challenging issues are the overlap of other forms of delirium in perioperative and intensive care settings as well as general anesthesia masking the onset of withdrawal symptoms. In contrast to other etiologies of delirium, the pathophysiology and thus treatment strategy of AWS is different: the key point is the tolerance to GABAergic molecules of alcohol-dependent subjects resulting in central nervous hyperactivity once the effect of alcohol or other GABA-stimulating agents is decreased. Summary: Despite limitations due to insufficient accuracy of self-reporting questionnaires and limited feasibility in emergency settings, the AUDIT and the shortened AUDIT-C are the standard tools for detection of alcohol use disorders (AUD), as well as predicting AWS risk and severity in approximately half of these AUD patients. The most important risk factors for AWS are a high blood alcohol concentration at hospital admission, AWS episodes in medical history, and lack of control of alcohol use. Patients considered
\end{abstract}

karger@karger.com www.karger.com/vis

Karger ${ }^{\prime \prime}=$
(C) 2020 The Author(s)

This article is licensed under the Creative Commons Attribution 40 International License (CC BY) (http://wwwlarger.com/Services/ OpenAccessLicense). Usage, derivative works and distribution are OpenAccessicense. Usage, derive original publisher. at risk for severe AWS must be treated with prophylactic medication before the onset of symptoms. Thiamine supplementation is required for all malnourished alcohol-dependent patients. Writing down alcohol-related diagnoses in the medical records requires the patient's presumed consent after shared decision-making. These reports should remain strictly confidential if the patient desires. Psychological support for the perioperative period as well as the following course should be offered to all AUD patients including support in short- and long-term detoxification. Alternative diagnoses must be ruled out with no timely delay, especially if fever and coma are the leading symptoms. The backbone of AWS therapy is the symptom-triggered administration of intravenous benzodiazepines (BZO) in escalating doses until the aimed revised Clinical Institute Withdrawal Assessment for Alcohol Scale (CIWA-Ar) or Richmond Agitation-Sedation Scale (RASS) score is achieved. Clonidine, dexmedetomidine, baclofen, ketamine, and neuroleptics may be used as symptom-orientated adjuncts. The therapeutic administration of ethanol or clomethiazole is considered to be harmful in critically ill patients after the onset of AWS. General supportive and intensive care including high-dose thiamine supplementation are mandatory in severe AWS cases. The timely differential diagnosis of delirium is important - and AWS is a diagnosis of exclusion - because BZO are strongly recommended for AWS patients but may not be the treatment of 
choice in other etiologies of delirium. Key Messages: Screening for AWS risk factors should be integrated in the preoperative and emergency assessment. Other severe diagnoses must be ruled out before the diagnosis of AWS can be established. Preventive treatment should be given to high-risk patients scoring positive for AUD and for patients with a lack of alcohol use control. The principles of AWS therapy are symptom-orientated doses of BZO and as adjuncts $a_{2}$-agonists, neuroleptics, and others guided by repeated reassessment with validated tools and thiamine administration. Length of stay and morbidity are reduced if AWS therapy is symptomorientated and protocol-based.

(c) 2020 The Author(s)

Published by S. Karger AG, Base

\section{Delirium and Its Differential Diagnosis: Alcohol Withdrawal Syndrome}

One in five surgical patients [1] has an alcohol use disorder (AUD). Especially in post-traumatic emergency surgery and in patients with aero-digestive oncology surgery, AUD are seen in $50 \%$ of the patients. Half of these, meaning approximately $25 \%$ of all patients in these surgical groups, have alcohol dependency [2]. The reported prevalence varies depending on the surgical specialty and the used screening methods.

In the perioperative setting, cognitive impairment after an invasive procedure is commonly called postoperative delirium (POD). A suggested causal explanation of POD is the concept of inflammation causing a transient complex neuronal dysfunction [3], but other mechanisms and factors are also relevant, e.g., perfusion, anemia/hypoxia, metabolic disorders, trauma, toxicity, and withdrawal. Alcohol-dependent patients not only have a higher risk of becoming delirious after surgical procedures [4], but also of developing other complications after surgery, such as infections, arrhythmias, and bleeding $[5,6]$.

The differential diagnosis of delirium includes alcohol withdrawal syndrome (AWS). The definition of AWS demands a timely association and suggests causality between alcohol intake reduction and onset of delirium symptoms. Thus, this is a typical situation when dependent patients are hospitalized, as abstinence is usually mandated by hospital settings.

The European Society of Anesthesiology describes hyperactive, hypoactive, and mixed forms of POD [4]. The precipitating etiology of POD is multifactorial including sedating drugs, inflammation, extended surgery, and acute complications as well as withdrawal. Predisposing factors, such as frailty and multimorbidity, are seen as patient-linked risk factors [7]. AWS symptoms are as unspecific as cognitive impairment, autonomous signs, tachycardia, vomiting, sweating, and fever and they occur after cessation or reduction of excessive alcohol use [8]. Severe cases with relevant mortality, often called "delirium tremens," are characterized by hallucinations, profound confusion, hypertension with tachycardia, and complicated seizures [9].

For AWS, there are established specific animal models in which the neuronal pathophysiology has been studied: Waller et al. [10] described a selectively bred population of rats that voluntarily drank large amounts of a $10 \%$ ethanol solution over several weeks and thus became alcohol dependent. These rats showed a hyperactive withdrawal syndrome for $72 \mathrm{~h}$ upon removal of ethanol. The severity of animal AWS was dependent on the duration and daily dose of alcohol ingestion [11]. The observed withdrawal symptoms were to a great extent similar to those of human AWS: a short phase of acute withdrawal (central hyperexcitability, autonomous hyperactivity, and motor abnormalities) was followed by a protracted phase of anxiety, sleep disturbances, and anhedonia [12]. In addition, different anesthetics seem to trigger different transmitter imbalances: in a rat model, animals with AWS exposed to desflurane anesthesia showed increased inhibitory amino acid arginine levels whereas $\gamma$-aminobutyric acid (GABA) levels decreased. This was not seen after sevoflurane anesthesia [13].

AWS is by definition a delirium, like all deliria a potentially lethal condition, initiating around 6-48 h after the last alcohol consumption and usually improving after 1 week. Underlying mechanisms are a decreased cerebral GABA-A receptor responsiveness as well as altered dopamine, glutamate, and norepinephrine feedback loops in the mesolimbic system [14]. AWS is known to be triggered by specific changes in neurophysiological pathways [12] developed under the long-term influence of regular alcohol intake. Abrupt removal of this central nervous depressant agent unmasks the tolerance to chronic alcohol intake and leads to symptoms of nervous hyperactivity.

The differential diagnosis of delirium with respect to AWS is important due to different approaches in the choice of therapeutic agents. Current reviews and guidelines recommend a benzodiazepine (BZO)-based prophylaxis and therapy for AWS patients [15], while they advise nonpharmacologic measures for POD or symptom-orientated approaches in sepsis-induced delirium, suggesting symptomatic non-BZO agents for alleviating hyperactive forms if anxiety is not present [16].

Another syndrome that overlaps with AWS in hospitalized alcohol-dependent patients is Wernicke's encephalopathy caused by nutritional thiamine deficiency [17]. The classic symptom triad of Wernicke's encephalopathy consists of ophthalmoplegia, ataxia, and confusion. Neuropathological correlates are lesions in the periventricular region, diencephalon, midbrain, hypothalamus, and 
cerebellar vermis. Autopsy studies suggest signs of Wernicke's encephalopathy in $0.4-2.8 \%$ of the population and in $12.5 \%$ of persons with AUD [18]. As in AWS, symptom variability and other overlapping manifestations of the primary hospitalization disease, such as traumatic brain injury, sepsis, or stroke, can complicate the diagnosis. Hence, Wernicke's encephalopathy is often underdiagnosed.

\section{Identifying Patients at Risk: Screening and Prophylaxis}

Screening of patients at risk of developing AWS is usually based on validated questionnaires about alcohol consumption habits and biomarkers [19]. For the perioperative setting, question-based self-reporting tests can detect AUD.

The Alcohol Use Disorders Identification Test (AUDIT) [20] is a 10-item questionnaire developed by the World Health Organization, designed for screening in primary health care settings. Each question has 3 or 5 possible answers, each reflecting $0-4$ points. Thus, the total score can vary between zero points for subjects that never consume alcohol and 40 points for most problematic drinking habits. Using the 8-point threshold, the test has shown a $92 \%$ sensitivity and $94 \%$ specificity for harmful alcohol use. Better suitable for clinical routine, the shortened AUDIT-C [21] consists of only three 4-point questions for frequency, typical amount, and binge-drinking habits: at least 4 out of 12 points for female and 5 for male subjects indicate a possible AUD with a sensitivity of $86 \%$ and a specificity of $72 \%$.

Before the AUDIT was promoted by extensive research, the CAGE questionnaire [22] was the most frequently reported screening tool. This test contains the following 4 questions: Have you ever felt you needed to cut down on your drinking? Have people annoyed you by criticizing your drinking? Have you ever felt guilty about drinking? Have you ever felt you needed a drink first thing in the morning (eye-opener) to steady your nerves or to get rid of a hangover? Abuse or dependence on alcohol is suggested by 2 positive answers, indicating further investigation is warranted [23].

While the CAGE test focuses on misuse and dependence symptoms, AUDIT also looks for frequency, amount, and sequelae of alcohol intake. Self-reporting tests can only identify patients at risk when the questions are answered properly. As drinking habits are usually underreported [24], laboratory findings can also be used to unmask a possible AUD. According to one systematic review [25], limited evidence suggests that among laboratory markers, a lower initial platelet count and lower serum potassium level were predictive of severe AWS, as well as higher initial alanine aminotransferase and serum $\gamma$-glutamyl transpeptidase. Newer forensic literature [26] also discusses fatty acid ethyl esters in hair and phosphatidylethanol in blood (specificity $48-89 \%$ and sensitivity 88-100\% for recent alcohol intake).

Combining clinical signs and surveys leads to a higher positive likelihood ratio for the prediction of severe AWS: the Luebeck Alcohol Withdrawal Risk Scale (LARS) [27], as well as its short version "LARS11", combine questions about medical history, actual blood alcohol concentration, and clinical signs. The calculated positive and negative predictive values were 76 and $98.7 \%$ for the prediction of severe AWS.

The first validated tool for the prediction of complicated AWS was the Prediction of Alcohol Withdrawal Severity Scale (PAWSS) [28]: patients with proven alcoholemia upon hospital admission or admitting to having drunk alcohol within the last month ("threshold criteria") were being interviewed by eight yes-no questions. The number of "yes" answers combined with 1 point each for a blood alcohol concentration above $200 \mathrm{mg} / \mathrm{dl}$ and increased autonomic activity led to a count of $0-10$ points. The authors found out that a PAWSS cutoff of 4 points identified patients developing complicated AWS with a sensitivity of $93.1 \%$ and a specificity of $99.5 \%$.

Despite the risks of AWS, the individual right to deny alcohol-drinking habits should be respected. It is ethically relevant to address AUD but only to document it with the presumed patient's consent after shared decisionmaking: AUD documentation in a medical report - even if only suspected - can have devastating consequences if files are accessed by others, e.g., family members, insurance companies, employers, or criminal prosecution organs. As with all psychiatric diagnoses, these medical reports must be treated with the utmost confidentiality due to still-existing stigmatization in our society. The most promising option is to evaluate AUD within an integrated lifestyle assessment and to intervene according to FRAMES (feedback, responsibility, advice, menu of behavioral change, empathy, self-efficiency), which is usable for emergency and elective settings [24, 29].

Due to the high morbidity and mortality of AWS, screening and prophylaxis in the perioperative setting are mandatory [2]: among individuals with a history of longterm heavy alcohol drinking, 50\% might develop AWS [30]; $90 \%$ of these cases are mild and easily managed by symptomatic treatment while the remaining $10 \%$ of the symptomatic patients develop generalized tonic-clonic seizures or delirium tremens [19]. Especially for severely alcohol-dependent subjects, it is ethically required to provide prophylactic AWS medication during the perioperative period as shown in a systematic review [15]: before the onset of AWS symptoms, BZO as well as continued oral or enterally applied alcohol administration in a dose 


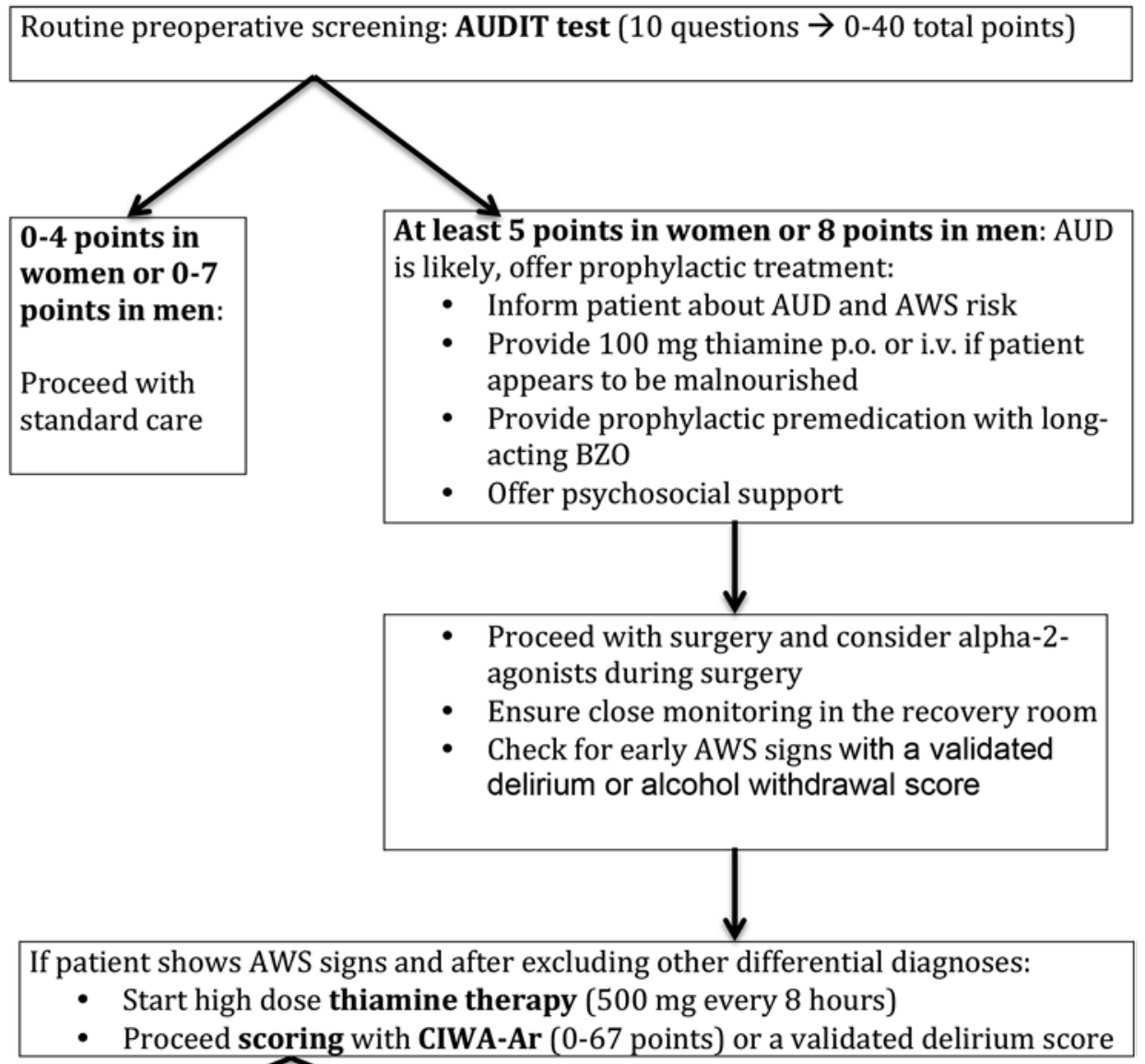

0-7 points: continue standard care including analgetics, antiemetics and delirium screening

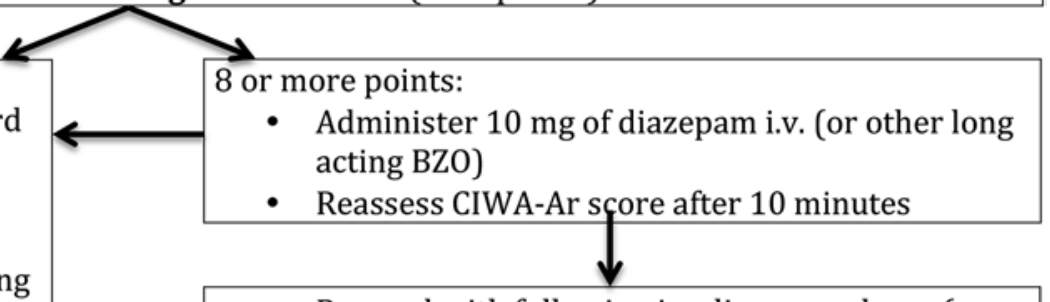

- Proceed with following i.v. diazepam doses (or other long acting BZO) after each reassessment if score remains positive (every 10 minutes):

$10 \mathrm{mg} \rightarrow 20 \mathrm{mg} \rightarrow 20 \mathrm{mg} \rightarrow 40 \mathrm{mg} \rightarrow \ldots$ (double the dose at every third administration if effect seems to be insufficient)

- Start symptom-oriented alpha-2-agonists for autonomous signs or low-dose haloperidol for

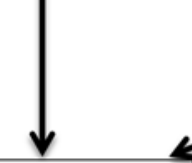
productive-psychotic signs ${ }^{1}$

Stabilized patients: Reassess CIWA-Ar every 8 hours until 48 hours after hospitalization

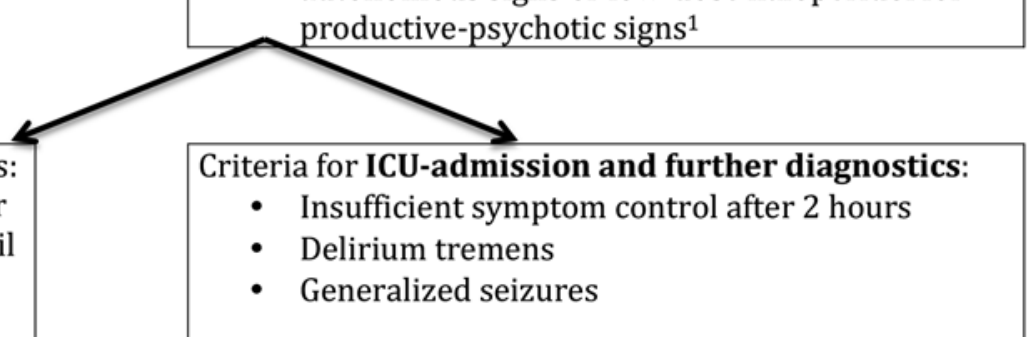

Fig. 1. Perioperative alcohol withdrawal syndrome: suggested algorithm for screening and therapy. AUD, alcohol use disorder; AUDIT, Alcohol Use Disorders Identification Test; AWS, alcohol withdrawal syndrome; BZO, benzodiazepine; CIWA-Ar, revised Clinical Institute Withdrawal Assessment for Alcohol Scale; ICU, intensive care unit; i.v., intravenous. ${ }^{1}$ See Spies and Rommelspacher [5]. 
of $0.5 \mathrm{~g} / \mathrm{kg}$ body weight/day are proven to be safe and effective in preventing severe withdrawal [31]. In addition, other concepts with adjuncts such as $\alpha_{2}$-agonists have been proven to be efficient [32].

Patients that screened positive for AUD should be offered psychosocial support for the perioperative period as well as for the postoperative phase, including psychosocial counseling and detoxification offers. This should be embedded in the local health care structures. In our department, we use the AUDIT for preoperative screening, at best embedded in a lifestyle assessment by a computerized version considering FRAMES criteria [24, 30], and offer counseling by a psychiatrist to each patient testing positive. This offer should be repeated after a resolved AWS episode, as this crucial experience may open the option for a motivational interviewing and guidance towards long-term detoxification programs.

If patients show a positive blood alcohol level at admission, the intended operation should be performed depending on the urgency: if postponing the procedure means a relevant outcome risk for the patient, we suggest performing the operation under prophylactic treatment with $\mathrm{BZO}$, as suggested in our flowchart (see Fig. 1). In unresponsive emergency patients, blood alcohol testing belongs to routine screening as part of the rapid investigation of frequent causes of coma. However, despite positive testing, other causes of emergency should be ruled out since AUD are often accompanied, e.g., by infections, sepsis, arrhythmias, congestive heart failure, liver damage with lowered blood glucose levels, and trauma with intracranial bleeding.

Another relevant point to consider is that anesthesiologists should be aware of cross-tolerance of alcohol with most sedating anesthetics, such as BZO, propofol, volatile anesthetics, and opioids, as also shown in animal models [12]. Endorphins are also decreased in patients with chronic AUD, therefore the analgesic requirement must be adapted $[33,34]$.

\section{Therapy of Suspected AWS in the Perioperative Setting}

The management of AWS in the perioperative period is dependent on the additional stress due to surgery, e.g., pain, infections, cardiac insufficiencies, metabolic derangements, or increased bleeding [5, 6]. This includes correcting metabolic and hemodynamic derangements, fluid and blood product deficits, as well as general supportive care, such as early nutrition. One crucial challenge is the different etiologies of delirium, as discussed above. As AWS remains a clinical diagnosis after ruling out all other different etiologies, a timely set-up is required [35].
One of the most suggested validated tools for AWS severity assessment is the revised Clinical Institute Withdrawal Assessment for Alcohol Scale (CIWA-Ar) score [36]. This score consists of nine items, each with up to 7 attributable points. The calculated score between zero and 67 points at each assessment can be used for repeated administration of AWS medication. The suggested threshold for repeated BZO administration is 8 points. An alternative score especially for mechanically ventilated patients is any validated delirium score $[4,16]$, taking into account the level of sedation using the Richmond Agitation-Sedation Scale (RASS), which is the gold standard for measurement of agitation or sedation [16]. The RASS score ranges from -5 (deep coma/unarousable) through 0 (calm and alert, usually aimed score for stable intensive care patients) up to +4 (combative against staff). Reassessments of CIWA-Ar or other validated delirium scores are advised at least every 8 hours but should be repeated every 5-10 min during intravenous drug titration.

As shown in several randomized trials (for review evidence of the following statements, see Ungur et al. [15]), long-acting BZO such as lorazepam or diazepam administered in escalating symptom-triggered doses are the standard backbone therapy of AWS. It has been reported that the duration of AWS and hospital length of stay (LOS) were shortened if the BZO administration was protocol-based, while CIWA-Ar was the diagnostic tool mostly used [37].

An appropriate initial AWS treatment protocol would include repetitive titration of 5-10 $\mathrm{mg}$ of diazepam or 2-4 $\mathrm{mg}$ of lorazepam every $10 \mathrm{~min}$ until the aimed score is achieved and doubling the administered dose every third time should the effect not appear to be relevant. Clinicians must be aware that the required doses for severe AWS can vary substantially within the first $24 \mathrm{~h}$ [38]. Ceiling effects of BZO administration and BZO toxicity must be considered, and therefore, adjunct treatment is required in patients with AWS without the adequate effect after BZO.

Clomethiazole is not indicated in critically ill patients due to bronchial secretion and thus elevated risk of pneumonia. Also, phenytoin is not advised for AWS seizures due to low effectivity. The use of levetiracetam and carbamazepine as anticonvulsants for AWS is not sufficiently evaluated in critically ill patients. $\gamma$-Hydroxy-butyric acid and neuroleptics are second-line options for therapy of autonomous or productive AWS symptoms, respectively $[5,39]$, but there are relevant concerns regarding side effects and safety.

The $\alpha$-agonist clonidine is advised for autonomous AWS symptoms [40]. For dexmedetomidine, no relevant advantage could be demonstrated in AWS therapy when compared to BZO treatment alone [41]. Two trials even suggested a trend to longer LOS if dexmedetomidine was 
added to BZO therapy [42], especially for AWS treatment in medical intensive care unit (ICU) patients [43]. However, in combination with propofol, patients treated in the ICU with severe AWS had a shorter time to extubation and ICU LOS [44].

The French literature, in particular, discusses the GABA-B receptor agonist baclofen for AWS therapy. Traditionally used as an antispasmodic in neurology, it is also proposed to treat acute AWS-related seizures or craving symptoms in alcohol-dependent persons [45]. Mechanisms of action are direct anti-epileptic effects of membrane hyperpolarization as well as activation of central GABA-B receptors involved in dopamine, serotonin, norepinephrine, and glutamate systems. Problematic points are the high dosage variability and the lack of highquality research data [46]. For gabapentin, more recent reviews report contradictory findings. However, a larger retrospective analysis [47] shows no benefit of additive gabapentin compared with only lorazepam therapy for AWS. Ketamine $0.15-0.3 \mathrm{mg} / \mathrm{kg} / \mathrm{h}$ was reported to be a useful adjunct to BZO in a small retrospective analysis [48], shortening LOS in intensive care.

Thiamine therapy is safe and results in rapid clinical improvement of acute Wernicke's encephalopathy [17]. Therapeutic dosage recommendations vary from $50 \mathrm{mg} /$ day for 3 days (US guidelines) up to $500 \mathrm{mg}$ every $8 \mathrm{~h}$ for 3-5 days followed by dosage tapering until clinical improvement (British and German guidelines) [18]. For prophylaxis, low-dose substitution is recommended for hospitalized AUD patients ( $100 \mathrm{mg} /$ day) if they appear to be malnourished, but not routinely for all asymptomatic AUD patients.

\section{Conclusions}

In emergency surgery, delirium requires a differential diagnosis and AWS can only be diagnosed if other etiologies are ruled out.
Screening for AWS risk factors should be standard during preoperative assessment. The care for high-risk patients during the first $48 \mathrm{~h}$ after cessation of alcohol intake must include prophylactic medication before the onset of AWS symptoms, intensive care monitoring, repeated assessment for AWS symptoms by validated scores, and thiamine prophylaxis for malnourished patients. Clinicians should be aware of the consequences of documenting alcohol-related diagnoses in medical reports.

Once AWS symptoms begin, a protocolized, symptom-orientated BZO-based therapy must be initiated. Differential diagnoses should be considered. The doses must be repeated and increased until symptoms are sufficiently controlled. Repeated CIWA-Ar or validated delirium assessments should be performed to guide treatment. Thiamine for symptomatic AWS patients is mandatory. Clonidine, baclofen, ketamine, and neuroleptics are symptom-orientated optional adjuncts.

\section{Disclosure Statement}

The authors have no specific conflicts of interest resulting from financial involvement. T. Neumann is a member of the Board of the German Society of Addiction Medicine (Deutsche Gesellschaft für Suchtmedizin). The first author's membership of the German branch of the "No Free Lunch" organization might lead to a critical rating of certain high-priced agents.

\section{Funding Sources}

All authors are employed by a public teaching hospital and wrote this manuscript as part of their regular work. No other funding sources are linked to this article.

\section{Author Contributions}

Alexander Lavinius Ungur und Claudia Spies: main text body, literature search, and final revision. Tim Neumann: major revisions and literature search. Friedrich Borchers: literature search and minor revisions.

\section{References}

1 Egholm JW, Pedersen B, Møller AM, Adami J, Juhl CB, Tønnesen H. Perioperative alcohol cessation intervention for postoperative complications. Cochrane Database Syst Rev. 2018 Nov;11:CD008343.

2 Kork F, Neumann T, Spies C. Perioperative management of patients with alcohol, tobacco and drug dependency. Curr Opin Anaesthesiol. 2010 Jun;23(3):384-90.

3 Vasunilashorn SM, Ngo L, Inouye SK, Libermann TA, Jones RN, Alsop DC, et al. Cytokines and postoperative delirium in older patients undergoing major elective surgery. J Gerontol A Biol Sci Med Sci. 2015 Oct;70(10):1289-95.

4 Aldecoa C, Bettelli G, Bilotta F, Sanders RD, Audisio R, Borozdina A, et al. European Soci- ety of Anaesthesiology evidence-based and consensus-based guideline on postoperative delirium. Eur J Anaesthesiol. 2017 Apr;34(4): 192-214.

5 Spies CD, Rommelspacher H. Alcohol withdrawal in the surgical patient: prevention and treatment. Anesth Analg. 1999 Apr;88(4): 946-54.

6 Spies CD, Nordmann A, Brummer G, Marks C, Conrad C, Berger G, et al. Intensive care unit stay is prolonged in chronic alcoholic men following tumor resection of the upper digestive tract. Acta Anaesthesiol Scand. 1996 Jul;40(6):649-56.

7 Fürstenau D, Spies C, Gersch M, Vogel A, Mörgeli R, Poncette AS, et al. Sharing frailty- related information in perioperative care: an analysis from a temporal perspective. BMC Health Serv Res. 2019 Feb;19(1):105.

8 National Clinical Guideline Centre (UK). Alcohol use disorders: diagnosis and clinical management of alcohol-related physical complications [Internet]. 2010. Available from: https:// ww w.ncbi.nlm.nih.gov/ pubmed/22876380.

9 Junghanns K, Wetterling T. [Alcohol withdrawal and its major complications]. Fortschr Neurol Psychiatr. 2017 Mar;85(3):163-77. German.

10 Waller MB, McBride WJ, Lumeng L, Li TK. Induction of dependence on ethanol by freechoice drinking in alcohol-preferring rats. Pharmacol Biochem Behav. 1982 Mar;16(3):501-7. 
11 Kampov-Polevoy AB, Matthews DB, Gause L, Morrow AL, Overstreet DH. P rats develop physical dependence on alcohol via voluntary drinking: changes in seizure thresholds, anxiety, and patterns of alcohol drinking. Alcohol Clin Exp Res. 2000 Mar;24(3):278-84.

12 Heilig M, Egli M, Crabbe JC, Becker HC. Acute withdrawal, protracted abstinence and negative affect in alcoholism: are they linked? Addict Biol. 2010 Apr;15(2):169-84.

13 Seidemann T, Spies C, Morgenstern R, Wernecke KD, Netzhammer N. Influence of volatile anesthesia on the release of glutamate and other amino acids in the nucleus accumbens in a rat model of alcohol withdrawal: a pilot study. PLoS One. 2017 Jan;12(1):e0169017.

14 Schuckit MA. Recognition and management of withdrawal delirium (delirium tremens). N Engl J Med. 2014 Nov;371(22):2109-13.

15 Ungur LA, Neuner B, John S, Wernecke K, Spies C. Prevention and therapy of alcohol withdrawal on intensive care units: systematic review of controlled trials. Alcohol Clin Exp Res. 2013 Apr;37(4):675-86.

16 Müller A, Weiß B, Spies CD, Leitliniengruppe S; S3-Leitliniengruppe. ["Symptomatic treatment of delirium, anxiety and stress, and protocol based analgesia, sedation and management of sleep in intensive care patients"]. Anasthesiol Intensivmed Notfallmed Schmerzther. 2015 Nov;50(11-12):698-703. German.

17 Zahr NM, Kaufman KL, Harper CG. Clinical and pathological features of alcohol-related brain damage. Nat Rev Neurol. 2011 May; 7(5):284-94.

18 Pruckner N, Baumgartner J, Hinterbuchinger B, Glahn A, Vyssoki S, Vyssoki B. Thiamine substitution in alcohol use disorder: a narrative review of medical guidelines. Eur Addict Res. 2019;25(3):103-10.

19 Wood E, Albarqouni L, Tkachuk S, Green CJ, Ahamad K, Nolan S, et al. Will this hospitalized patient develop severe alcohol withdrawal syndrome?: The Rational Clinical Examination Systematic Review. JAMA. 2018 Aug 320(8):825-33.

20 Conigrave KM, Hall WD, Saunders JB. The AUDIT questionnaire: choosing a cut-off score. Alcohol Use Disorder Identification Test. Addiction. 1995 Oct;90(10):1349-56.

21 Conigrave KM, Saunders JB, Reznik RB. Predictive capacity of the AUDIT questionnaire for alcohol-related harm. Addiction. 1995 Nov;90(11):1479-85.

22 Ewing JA. Detecting alcoholism. The CAGE questionnaire. JAMA. 1984 Oct;252(14): 1905-7.

23 Awissi DK, Lebrun G, Coursin DB, Riker RR, Skrobik Y. Alcohol withdrawal and delirium tremens in the critically ill: a systematic review and commentary. Intensive Care Med. 2013 Jan;39(1):16-30.

24 Kip MJ, Neumann T, Jugel C, Kleinwaechter R, Weiss-Gerlach E, Guill MM, et al. New strategies to detect alcohol use disorders in the preoperative assessment clinic of a German university hospital. Anesthesiology. 2008 Aug;109(2):171-9.
25 Goodson CM, Clark BJ, Douglas IS. Predictors of severe alcohol withdrawal syndrome: a systematic review and meta-analysis. Alcohol Clin Exp Res. 2014 Oct;38(10):2664-77.

26 Andresen-Streichert H, Müller A, Glahn A, Skopp G, Sterneck M. Alcohol biomarkers in clinical and forensic contexts. Dtsch Arztebl Int. 2018 May;115(18):309-15.

27 Wetterling $\mathrm{T}$, Weber $\mathrm{B}$, Depfenhart $\mathrm{M}$, Schneider B, Junghanns K. Development of a rating scale to predict the severity of alcohol withdrawal syndrome. Alcohol Alcohol. 2006 Nov-Dec;41(6):611-5.

28 Maldonado JR, Sher Y, Das S, Hills-Evans K, Frenklach A, Lolak S, et al. Prospective validation study of the Prediction of Alcohol Withdrawal Severity Scale (PAWSS) in medically ill inpatients: a new scale for the prediction of complicated alcohol withdrawal syndrome. Alcohol Alcohol. 2015 Sep;50(5): 509-18.

29 Neumann T, Neuner B, Weiss-Gerlach E, Tønnesen H, Gentilello LM, Wernecke KD, et al. The effect of computerized tailored brief advice on at-risk drinking in subcritically injured trauma patients. J Trauma. 2006 Oct; 61(4):805-14.

30 Schmidt KJ, Doshi MR, Holzhausen JM, Natavio A, Cadiz M, Winegardner JE. Treatment of severe alcohol withdrawal. Ann Pharmacother. 2016 May;50(5):389-401.

31 Eggers V, Tio J, Neumann T, Pragst F, Müller C, Schmidt LG, et al. Blood alcohol concentration for monitoring ethanol treatment to prevent alcohol withdrawal in the intensive care unit. Intensive Care Med. 2002 Oct; 28(10):1475-82.

32 Spies CD, Dubisz N, Funk W, Blum S, Müller $\mathrm{C}$, Rommelspacher H, et al. Prophylaxis of alcohol withdrawal syndrome in alcohol-dependent patients admitted to the intensive care unit after tumour resection. Br J Anaesth. 1995 Dec;75(6):734-9.

33 Spies C, Eggers V, Szabo G, Lau A, von Dossow $\mathrm{V}$, Schoenfeld $\mathrm{H}$, et al. Intervention at the level of the neuroendocrine-immune axis and postoperative pneumonia rate in long-term alcoholics. Am J Respir Crit Care Med. 2006 Aug;174(4):408-14

34 Rosenberger P, Mühlbauer E, Weissmüller T, Rommelspacher H, Sinha P, Wernecke KD, et al. Decreased proopiomelanocortin mRNA in lymphocytes of chronic alcoholics after intravenous human corticotropin releasing factor injection. Alcohol Clin Exp Res. 2003 Nov; 27(11):1693-700.

35 Mayo-Smith MF, Beecher LH, Fischer TL, Gorelick DA, Guillaume JL, Hill A, et al.; Working Group on the Management of Alcohol Withdrawal Delirium, Practice Guidelines Committee, American Society of Addiction Medicine. Management of alcohol withdrawal delirium. An evidence-based practice guideline. Arch Intern Med. 2004 Jul;164(13): 1405-12.
36 Sullivan JT, Sykora K, Schneiderman J, Naranjo CA, Sellers EM. Assessment of alcohol withdrawal: the revised Clinical Institute Withdrawal Assessment for Alcohol Scale (CIWAAr). Br J Addict. 1989 Nov;84(11):1353-7.

37 Spies CD, Otter HE, Hüske B, Sinha P, Neumann T, Rettig J, et al. Alcohol withdrawal severity is decreased by symptom-orientated adjusted bolus therapy in the ICU. Intensive Care Med. 2003 Dec;29(12):2230-8.

38 DeBellis R, Smith BS, Choi S, Malloy M. Management of delirium tremens. J Intensive Care Med. 2005 May-Jun;20(3):164-73.

39 Lenzenhuber E, Müller C, Rommelspacher H, Spies C. [Gamma-hydroxybutyrate for treatment of alcohol withdrawal syndrome in intensive care patients. A comparison between with two symptom-oriented therapeutic concepts]. Anaesthesist. 1999 Feb;48(2):89-96. German.

40 Spies CD, Dubisz N, Neumann T, Blum S, Müller C, Rommelspacher $\mathrm{H}$, et al. Therapy of alcohol withdrawal syndrome in intensive care unit patients following trauma: results of a prospective, randomized trial. Crit Care Med. 1996 Mar;24(3):414-22.

41 Beg M, Fisher S, Siu D, Rajan S, Troxell L, Liu VX. Treatment of alcohol withdrawal syndrome with and without dexmedetomidine. Perm J. 2016;20(2):49-53.

42 VanderWeide LA, Foster CJ, MacLaren R, Kiser TH, Fish DN, Mueller SW. Evaluation of early dexmedetomidine addition to the standard of care for severe alcohol withdrawal in the ICU: a retrospective controlled cohort study. J Intensive Care Med. 2016 Mar; 31(3):198-204.

43 Mueller SW, Preslaski CR, Kiser TH, Fish DN, Lavelle JC, Malkoski SP, et al. A randomized, double-blind, placebo-controlled dose range study of dexmedetomidine as adjunctive therapy for alcohol withdrawal. Crit Care Med. 2014 May;42(5):1131-9.

44 Love K, Zimmermann AE. Use of propofol plus dexmedetomidine in patients experiencing severe alcohol withdrawal in the intensive care unit. J Clin Pharmacol. 2020 Apr;60(4): 439-443.

45 de Beaurepaire R. A review of the potential mechanisms of action of baclofen in alcohol use disorder. Front Psychiatry. 2018 Oct;9: 506.

46 Cooney G, Heydtmann M, Smith ID. Baclofen and the alcohol withdrawal syndrome - a short review. Front Psychiatry. 2019 Jan;9: 773.

47 Andaluz A, DeMoss D, Claassen C, Blair S, Hsu J, Bakre S, et al. Fixed-dose gabapentin augmentation in the treatment of alcohol withdrawal syndrome: a retrospective, openlabel study. Am J Drug Alcohol Abuse. 2020; 46(1):49-57.

48 Pizon AF, Lynch MJ, Benedict NJ, Yanta JH, Frisch A, Menke NB, et al. Adjunct ketamine use in the management of severe ethanol withdrawal. Crit Care Med. 2018 Aug; 46(8):e768-71. 